\title{
Pseudoclassical theories of Majorana, Weyl and Majorana-Weyl particles
}

\author{
Grigoryan G.V.`, Grigoryan R.P. ${ }^{\star \star}$, Tyutin I.V.†⿴囗十
}

Yerevan Physics Institute, Republic of Armenia

\author{
^E-mail:GAGRI@VXC.YERPHY.AM \\ ^^E-mail:ROGRI@VXC.YERPHY.AM \\ $\ddagger$ E-mail:TYUTIN@LPI.AC.RU
}

\begin{abstract}
A pseudoclassical theories of Majorana, Weyl and Majorana-Weyl particles in the spacetime dimensions $D=2 n$ are constructed. The canonical quantization of these theories is carried out and as a result we obtain the quantum mechanical description of neutral particle in $D=2 n$, Weyl particle in $D=2 n$ and neutral Weyl particle in $D=4 n+2$. In $D=2,4(\bmod 8)$ dimensional space-time the description of the neutral particle coincides with the field theoretical description of the Majorana particle in the Foldy-Wouthuysen representation. In $D=8 k+2$ dimensions the neutral Weyl particle coincides with the Majorana-Weyl particle in the Foldy-Wouthuysen representation.
\end{abstract}

\section{Yerevan Physics Institute Yerevan 1995}

\footnotetext{
* P.N.Lebedev Physical Institute, Moscow, Russia

$\dagger$ Partially supported by the grant 211-5291 YPI of the German Bundesministerium für Forschung und Technologie.
} 


\section{Introduction}

In spite of the bulk of the papers devoted to the theories of point particles and to methods of their quantization, the problem still attracts the attention of the investigators and numerous classical models of particles and superparticles were discussed recently. The renewal of the interest to these theories is primarily due to the problems in the string theory since particles are prototypes of the strings.

The first pseudoclassical description of the relativistic spinning particle was given in paper [1, 2]. It was followed by a great number of papers [3-22] devoted to the different quantization schemes of that theory, to the introduction of the internal symmetries and to the generalization to higher spins.

A pseudoclassical theory of Weyl particle in the space-time $D=4$ was constructed in [23]. Their method was used in [24] to construct the theory of Weyl particle in the space-time dimensions $D=2 n$, where a quantum mechanics of the neutral Weyl particle

in even-dimensional space-time was suggested and the connection of this theory with the theory of Majorana-Weyl particle in QFT for $D=10$ was discussed.

In this paper the description of Majorana, Weyl and Majorana-Weyl particles in the pseudoclassical approach is investigated in arbitrary even dimensions. This is interesting in connection with the following.

As it is known, the theory of RNS string with a GSO projection (see. 225) is a supersymmetric theory in ten dimensional space-time. The supersymmetry requires that each mass level comprises a supermultiplete. In the massless sector the superpartners to the gauge vector fields, which in $D=10$ dimensions have eight degrees of freedom, are massless fermions - Majorana-Weyl bispinors. Hence it is interesting to construct the classical (pseudoclassical) model, which after quantization will bring to the theory of the Majorana-Weyl bispinor in $D=10$ dimensions.

In section 2 the pseudoclassical theory of $D=2 n$ dimensional neutral spinning particle is presented. The canonical quantization of that theory in arbitrary even dimensional space-time is carried out and it's found, that for $D=2,4(\bmod 8)$ the quantum mechanical description coincides with the field theoretical description of the Majorana particle in 
the Foldy-Wouthuysen representation. In section 3 the pseudoclassical theory of Weyl particle in $D=2 n$ dimensional space-time is investigated. The canonical quantization of that theory results in the theory which coincides with field theoretical description of the $D=2 n$ dimensional Weyl particle in the Foldy-Wouthuysen representation. The section 4 is devoted to the canonical quantization of the neutral Weyl particle in the spacetime dimensions $D=4 n+2$ and it is shown that in the dimensions $D=8 k+2$ this theory coincides with the theory of the Majorana-Weyl particle in the Foldy-Wouthuysen representation in the field theory.

\section{Even dimensional neutral (Majorana) particle}

The quantum mechanical description of Majorana particle repeats in essence the similar description of the Dirac particle. The canonical quantization of the corresponding pseudoclassical theory of the Dirac particle [1] was carried out in [16] in the dimensions $D=4$ and in [17] in the arbitrary even dimensions $D=2 n$. We will write down the main important formulae, which will be used below ( for details see 116, 17]). The action of the theory is given by the expression

$$
S=\frac{1}{2} \int d \tau\left[\frac{1}{e}\left(\dot{x}^{\mu}-\frac{i}{2} \chi \xi^{\mu}\right)^{2}+e m^{2}+i m \chi \xi_{D+1}-i\left(\xi_{\mu} \dot{\xi}^{\mu}-\xi_{D+1} \dot{\xi}_{D+1}\right)\right] .
$$

Here $x^{\mu}$ are the coordinates of the particle, $\mu=0,1, \ldots, D-1 ; \xi^{\mu}$ are Grassmann variables describing the spin degrees of freedom; $e, \chi, \xi_{D+1}$ are additional fields, $e$ being Grassmann even, $\chi, \xi_{D+1}$-Grassmann odd variables; the overdote denotes a differentiation with respect to the parameter $\tau$ along the trajectory.

This action is invariant under two types of gauge transformations: the reparametrization transformations

$$
\begin{aligned}
& \delta x^{\mu}=u \dot{x}^{\mu}, \quad \delta e=\frac{d}{d \tau}(u e), \\
& \delta \xi^{\mu}=u \dot{\xi}^{\mu}, \quad \delta \xi_{D+1}=u \dot{\xi}_{D+1}, \quad \delta \chi=\frac{d}{d \tau}(u \chi)
\end{aligned}
$$

with the even parameter $u(\tau)$, and the supergauge transformations

$$
\delta x^{\mu}=i v \xi^{\mu}, \quad \delta e=i v \chi, \quad \delta \chi=2 \dot{v},
$$




$$
\delta \xi^{\mu}=v\left(\frac{\dot{x}^{\mu}}{e}-\frac{i \chi}{2 e} \xi^{\mu}\right), \quad \delta \xi_{D+1}=v m
$$

with the odd parameter $v(\tau)$.

The hamiltonization of the theory brings to the canonical hamiltonian

$$
H=\frac{e}{2}\left(p^{2}-m^{2}\right)+\frac{i}{2} \chi\left(p_{\mu} \xi^{\mu}-m \xi_{D+1}\right)
$$

and to the set of primary constraints

$$
\begin{aligned}
\Phi_{\mu} & \equiv \pi_{\mu}-\frac{i}{2} \xi_{\mu} \approx 0, \quad \mu=0,1, \ldots, D-1, \\
\Phi_{D} & \equiv \pi_{D+1}+\frac{i}{2} \xi_{D+1} \approx 0, \quad \Phi_{D+5} \equiv \pi_{e} \approx 0, \quad \Phi_{D+7} \equiv \pi_{\chi} \approx 0 .
\end{aligned}
$$

Here $p_{\mu}, \pi_{e}, \pi_{\mu}, \pi_{D+1}, \pi_{\chi}$ are canonical momenta conjugated to $x^{\mu}, e, \xi^{\mu}, \xi_{D+1}, \chi$ correspondingly. Using the Dirac procedure 26, 27] we find the secondary constraints

$$
\Phi_{D+1} \equiv p_{\mu} \xi^{\mu}-m \xi_{D+1} \approx 0, \quad \Phi_{D+3} \equiv\left|p_{0}\right|-\omega \approx 0 ; \quad \omega=\left(p_{i}^{2}+m^{2}\right)^{1 / 2}
$$

The theory contains first class constraints $\Phi_{D+3}, \quad \Phi_{D+5}, \quad \Phi_{D+7}, \quad$ and

$$
\varphi=p^{\mu} \Phi_{\mu}+m \Phi_{D}+i \Phi_{D+1}=\frac{1}{2} i\left(p^{\mu} \xi_{\mu}-m \xi_{D+1}\right)+p^{\mu} \pi_{\mu}+m \pi_{D+1}
$$

The additional gauge conditions, equal in number to that of all first class constraints and conjugated to the latter, which will fix the gauges of the theory, are chosen in the form:

$$
\begin{aligned}
\Phi_{D+4} \equiv & x^{0}-\kappa \tau \approx 0, \quad \Phi_{D+8} \equiv \chi \approx 0, \quad \Phi_{D+6} \equiv e+1 /\left|p_{0}\right| \approx 0 \\
& \Phi_{D+2} \equiv \xi^{0} \approx 0 ; \quad \kappa=-\operatorname{sign} p_{0} .
\end{aligned}
$$

After the canonical transformation

$$
x^{0} \rightarrow x^{0 \prime}=x^{0}-\kappa \tau
$$

(the rest of the variables remain unchanged), bringing to constraints which do not depend of time explicitly, the theory is described by the Hamiltonian (primes are omitted)

$$
H=\omega=\left(p_{i}^{2}+m^{2}\right)^{1 / 2}
$$


and by the independent variables $x^{i}, p_{i}, \xi^{i}$, for which the Dirac brackets for complete set of constraints have the form:

$$
\begin{aligned}
& \left\{x^{i}, x^{j}\right\}_{D}=\frac{i}{2 \omega^{2}}\left[\xi^{i}, \xi^{j}\right]_{-}, \quad\left\{x^{i}, p_{j}\right\}_{D}=\delta_{j}^{i}, \quad\left\{p_{i}, p_{j}\right\}_{D}=0 \\
& \left\{x^{i}, \xi^{j}\right\}_{D}=\frac{1}{\omega^{2}} \xi^{i} p^{j}, \quad\left\{\xi^{i}, \xi^{j}\right\}_{D}=-i\left(\delta^{i j}-\frac{p^{i} p^{j}}{\omega^{2}}\right), \quad\left\{p_{i}, \xi^{j}\right\}_{D}=0 .
\end{aligned}
$$

The quantization of the theory is carried out through the realization of the operators $\hat{x}^{i}, \hat{p}_{i}, \hat{\xi}^{i}$ in the form:

$$
\begin{aligned}
& \hat{x}^{i}=\hat{q}^{i}-\frac{i \hbar}{4} \frac{1}{\hat{\omega}(\hat{\omega}+m)}\left[\Sigma^{i}, \hat{p}_{j} \Sigma^{j}\right], \\
& \hat{\xi}^{i}=\left(\frac{\hbar}{2}\right)^{1 / 2} \hat{\kappa}\left[\Sigma^{i}-\frac{1}{\hat{\omega}(\hat{\omega}+m)} \hat{p}_{i} \hat{p}_{j} \Sigma^{j}\right], \quad \hat{p}_{k}=-i \frac{\partial}{\partial q^{k}},
\end{aligned}
$$

where operators $\hat{q}^{i}$ (physical coordinate operators) are multiplication operators, the variable $\kappa$ is replaced by the operator $\hat{\kappa}$

$$
\hat{\kappa}=\left(\begin{array}{cc}
I & 0 \\
0 & -I
\end{array}\right)=\tau^{3} \otimes I, \quad \hat{\kappa}^{2}=1
$$

with eigenvalues $\kappa= \pm 1$, the operators $\Sigma^{i}=\operatorname{diag}\left(\sigma^{i}, \sigma^{i}\right), \sigma^{i}$ are $2^{\frac{D-2}{2}} \times 2^{\frac{D-2}{2}}$ matrices, which realize the Clifford algebra $\left[\sigma^{i}, \sigma^{j}\right]_{+}=2 \delta^{i j} I$.

The wave function $f$ is given by

$$
f=\left(\begin{array}{l}
f^{1}(q) \\
f^{2}(q)
\end{array}\right)
$$

where $f^{1}$ and $f^{2}$ are $2^{\frac{D-2}{2}}$ component columns, and the Shrödinger equation has the form

$$
\left(i \partial / \partial x^{0}-\hat{\kappa} \hat{\omega}\right) f=0, \quad q=\left(x^{0}, q^{i}\right)
$$

where we passed from the variable $\tau$ to the the physical time $x^{0}=\kappa \tau$.

The canonical generators of the Lorentz transformation

$$
J^{\mu \nu}=-\left(x^{\mu} p^{\nu}-x^{\nu} p^{\mu}+\frac{i}{2}\left[\xi^{\mu}, \xi^{\nu}\right]_{-}\right)
$$

after quantization of the theory, in terms of operators of the physical variables, are given by the expressions

$$
\begin{aligned}
\hat{J}^{i k} & =-\hat{q}^{i} \hat{p}^{k}+\hat{q}^{k} \hat{p}^{i}-\frac{i \hbar}{4}\left[\Sigma^{i}, \Sigma^{k}\right]_{-}, \\
\hat{J}^{0 k} & =-x^{0} \hat{p}^{k}-\frac{1}{2} \hat{\kappa}\left[\hat{q}^{k}, \hat{\omega}\right]_{+}-\frac{i \hbar}{4 \hat{\omega}} \hat{\kappa} \hat{p}^{j}\left[\Sigma^{k}, \Sigma^{j}\right]_{-} .
\end{aligned}
$$


In [16, 17] it is shown, that $f^{1}$ must be interpreted as the wave function of the particle, $f^{2}$ as the complex conjugated wave function of the antiparticle and the quantum mechanical description coincides with the description of the Dirac particle in the field theory in the Foldy-Wouthuysen representation.

Lets turn now to the construction of the quantum mechanics of the Majorana particle. Note, that the action (1) is invariant under the transformations

$$
\begin{aligned}
x^{\mu}(\tau) & \rightarrow x^{\mu}(-\tau), \quad \xi^{\mu}(\tau) \rightarrow \xi^{\mu}(-\tau), \quad \xi_{D+1}(\tau) \rightarrow-\xi_{D+1}(-\tau), \\
\chi(\tau) & \rightarrow \chi(-\tau), \quad e(\tau) \rightarrow e(-\tau), \quad i \rightarrow-i,
\end{aligned}
$$

which correspond to the reparametrization $\tau \rightarrow-\tau$. This transformation was not included in the gauge group at the beginning of this section. In that case the model describes the charged particle and in the gauge $x^{0}-\kappa \tau \approx 0$ the trajectories with $\kappa=+1$ are interpreted as trajectories of particles and those with $\kappa=-1$ as trajectories of antiparticles. The switching on of the external electromagnetic field confirms this assertion since to the trajectory with a given $\kappa$ corresponds a particle with a charge $\kappa e$ [16] and the action isn't invariant under the transformation $\tau \rightarrow-\tau$. When the action is invariant under the transformation $\tau \rightarrow-\tau$, there is a possibility of another interpretation. We can identified the trajectories with $\kappa=+1$ and $\kappa=-1$. This is equivalent to including of the reparametrization $\tau \rightarrow-\tau$ in the gauge group [16] and then the theory describes the truly neutral particle.

The quantization of the theory in this case may be carried out in two ways:

A) to choose a gauge, which violates the reparametrization symmetry $\tau \rightarrow-\tau$ as well;

B) to quantize the theory in the gauge which doesn't violate the reparametrization symmetry $\tau \rightarrow-\tau$ and then factorize with respect to that symmetry (i.e. identify the trajectories with $\kappa=+1$ and $\kappa=-1$ ).

A) In this case the convenient choice of the gauge is the replacement of the constraint $\Phi_{D+4}$ by the constraint

$$
\Phi_{D+4}^{\prime}=x^{0}-\tau \approx 0 .
$$

The quantum mechanics in this case coincides with the sector $\kappa=+1$ of the theory 
described at the beginning of this section. In particular the wave function $f$ is now a $2^{\frac{D-2}{2}}$ component column, which coincides with the $f^{1}$ :

$$
f=f^{1}
$$

For the comparison of this theory with the description of the neutral (Majorana) particle in the field theory it is convenient to introduce the "bispinor" $f_{M}$

$$
f_{M}=\left(\begin{array}{c}
f^{1} \\
\Lambda f^{1 *}
\end{array}\right)
$$

The choice of the matrix $\Lambda$ will be discussed below.

B) The factorization with respect to the symmetry $\tau \rightarrow-\tau$ of the quantum mechanics of the Dirac particle consists in fact in the identification of the sectors $\kappa=+1$ and $\kappa=-1$. To give the rule of such identification note, that from the Shrödinger equation (15) it follows, that $f^{1}$ contains only positive frequencies, while $f^{2}$ contains only negative ones. Thus the rule must be of the form: $f^{2} \sim f^{1 *}$. This is in accordance with the interpretation of $f^{2}$ as a complex conjugated wave function of the antiparticle and also with the explicit form of the transformation $\tau \rightarrow-\tau$. Hence the factorization rule means, that in the state space of the Dirac particle we must restrain ourselves to the vectors having the form (21), where $\Lambda$ is a certain unitary operator, which we'll chose in the form of the numerical unitary $2^{\frac{D-2}{2}} \times 2^{\frac{D-2}{2}}$ matrix. Since $f^{1}$ and $f^{2}$ have definite transformation properties under Lorentz transformations, the $\Lambda$ matrix must satisfy the relation

$$
\hat{J}^{\mu \nu}(\kappa=-1)=-\Lambda \hat{J}^{\mu \nu *}(\kappa=+1) \Lambda^{+}
$$

Using the explicit form (17) of the $J^{\mu \nu}$ generators we find, that $\Lambda$ matrix must have the property

$$
\left[\sigma^{i}, \sigma^{j}\right]_{-}=\Lambda\left[\sigma^{i *}, \sigma^{j *}\right]_{-} \Lambda^{+}
$$

whence it follows that

$$
\sigma^{i}=\epsilon \Lambda \sigma^{i *} \Lambda^{+}, \quad \epsilon=+1 \text { or }-1
$$

It's not difficult to check, that if such a matrix exists, then it is unique up to a sign. We'll give the explicit expressions for the $\Lambda$ matrices in any even dimensional space-time $D=2 n$ (though the $\Lambda$ matrix exists in any dimensions). 
We'll choose a special form of the $\Gamma_{(2 n)}^{\mu_{(2 n)}}, \mu_{(2 n)}=0,1, \ldots, 2 n-1$ in the $D=2 n$ dimensions in the Dirac representation, which we'll describe inductively. Let by definition $\Gamma_{(0)}^{\mu_{(0)}}=1$. The $2 \times 2$ dimensional matrices $\Gamma_{(2)}^{\mu_{(2)}}$ are equal to

$$
\Gamma_{(2)}^{0}=\tau^{3}, \quad \Gamma_{(2)}^{1}=i \tau^{2}
$$

where $\tau^{k}, k=1,2,3$ are Pauli matrices. If the matrices $\Gamma_{(2 n)}^{\mu_{(2 n)}}$ are known, then the $\Gamma_{(2 n+2)}^{\mu_{(2 n+2)}}$ matrices are obtained by the rule

$$
\Gamma_{(2 n+2)}^{0}=\tau^{3} \otimes I_{(2 n)}, \quad \Gamma_{(2 n+2)}^{i}=i \tau^{2} \otimes \sigma_{(2 n+2)}^{i}, \quad i=1,2, \ldots, 2 n+1,
$$

where $I_{(2 n)}$ is a $2^{n} \times 2^{n}$ dimensional unit matrix, $2^{n} \times 2^{n}$ dimensional $\sigma_{(2 n+2)}^{i}$ matrices are given by

$$
\sigma_{(2 n+2)}^{1}=\Gamma_{(2 n)}^{D+1}, \quad \sigma_{(2 n+2)}^{k}=-i \Gamma_{(2 n)}^{k-1}, \quad k=2, \ldots, 2 n, \quad \sigma_{(2 n+2)}^{2 n+1}=\Gamma_{(2 n)}^{0},
$$

where $\Gamma^{D+1}$-matrix is equal to

$$
\Gamma_{(2 n)}^{D+1}=i^{n-1} \Gamma_{(2 n)}^{0} \cdots \Gamma_{(2 n)}^{2 n-1}=\tau^{1} \otimes I_{2 n-2} .
$$

Then the $\Lambda \equiv \Lambda_{(2 n)}$ is equal to

$$
\begin{aligned}
& \Lambda_{(2)}=1, \\
& \Lambda_{(4 k+2)}=\underbrace{\left(I_{(2)} \otimes \tau^{2}\right) \times \cdots \times\left(I_{(2)} \otimes \tau^{2}\right)}_{k \text { times }}=(-1)^{k} \Lambda_{(4 k+2)}^{T}=(-1)^{k} \Lambda_{(4 k+2)}^{*}=I_{(2)} \otimes \Lambda_{(4 k)}, \\
& \Lambda_{(4 k)}=\tau^{2} \otimes \underbrace{\left(I_{(2)} \otimes \tau^{2}\right) \times \cdots \times\left(I_{(2)} \otimes \tau^{2}\right)}_{k-1 \text { times }}=(-1)^{k} \Lambda_{(4 k)}^{T}=(-1)^{k} \Lambda_{(4 k)}^{*}=\tau^{2} \otimes \Lambda_{(4 k-2)},
\end{aligned}
$$

and

$$
\varepsilon \equiv \varepsilon_{(2 n)}=(-1)^{n+1}
$$

Thus in any even dimensional space-time we have a quantum mechanical description of the neutral "spin 1/2" particle, which is obtained by quantization of the pseudoclassical theory with the enlarged reparametrization gauge group.

Lets compare this theory with the description of the neutral (Majorana) particle in the field theory. In the Dirac representation the Majorana bispinor $\psi_{M}$ is defined by relation

$$
\psi_{M}=B_{F T} \psi_{M}^{*}, \quad B_{F T}=C \Gamma^{0}
$$


where $C$ is the charge conjugation matrix. As it is known 28 in the field theory this definition of the Majorana spinor is not contradictory only when the dimension of the space-time is $D=2,4(\bmod 8)$. In that case $B$ is a symmetric matrix.

In the quantum mechanics constructed above the wave function $f_{M}$ satisfies a similar condition in any dimensions:

$$
f_{M}=B_{Q M} f_{M}^{*}, \quad B_{Q M}=\left(\begin{array}{cc}
0 & \Lambda^{T} \\
\Lambda & 0
\end{array}\right) .
$$

To compare matrices $B_{F T}$ and $B_{Q M}$ note, that the quantum mechanical description corresponds to the Foldy-Wouthuysen representation in the field theory. Thus we must first turn from the Dirac representation to the Foldy-Wouthuysen representation . The $B_{F T}$ can be easily constructed explicitly. Note, that the inductive construction of $\Gamma$ matrices presented above ensures the properties of the latter:

$$
\Gamma_{(2 n)}^{0}=\Gamma_{(2 n)}^{0 T}=\Gamma_{(2 n)}^{0+}, \quad \Gamma_{(2 n)}^{i}=(-1)^{i} \Gamma_{(2 n)}^{i T}=-\Gamma_{(2 n)}^{i+}, \quad i=1, \ldots, 2 n-1
$$

These allow to explicitly construct the charge conjugation matrices $C_{(2 n)}$ by the rule

$$
C_{(2 n)}=\beta_{2 n} \prod_{k} \Gamma_{(2 n)}^{k}
$$

where $k$ are even for even $n$ and $k$ are odd for odd $n$ :

$$
\begin{aligned}
C_{(8 k)} & =\beta_{8 k} i(-1)^{k} \tau^{1} \otimes \Lambda_{(8 k)} \\
C_{(8 k+2)} & =\beta_{8 k+2} i(-1)^{k} \Lambda_{(8 k+4)} \\
C_{(8 k+4)} & =\beta_{8 k+4}(-1)^{k} \tau^{1} \otimes \Lambda_{(8 k+4)} \\
C_{(8 k+6)} & =\beta_{8 k+6}(-1)^{k} \Lambda_{(8(k+1))}
\end{aligned}
$$

The choice of the $\beta_{2 n}$ is a matter of convenience.

Using (35), the expression for $\Gamma^{0}$ matrice and the relations (29), we find

$$
\begin{aligned}
B_{F T(8 k)} & =\beta_{8 k}(-1)^{k} \tau^{2} \otimes \Lambda_{(8 k)} \\
B_{F T(8 k+2)} & =\beta_{8 k+2}(-1)^{k+1} \tau^{1} \otimes \Lambda_{(8 k+2)} \equiv \beta_{8 k+2}(-1)^{k+1} B_{Q M(8 k+2)} \\
B_{F T(8 k+4)} & =\beta_{8 k+4} i(-1)^{k+1} \tau^{2} \otimes \Lambda_{(8 k+4)} \equiv \beta_{8 k+4}(-1)^{k} B_{Q M(8 k+4)} \\
B_{F T(8 k+6)} & =\beta_{8 k+6} i(-1)^{k} \tau^{1} \otimes \Lambda_{(8 k+6)} .
\end{aligned}
$$


Turning to the Foldy-Wouthuysen representation, the $B_{F T}$ must be replaced by the matrices

$$
B_{F T} \rightarrow B_{F W}=U B_{F T} U^{T}
$$

where the unitary matrix $U$ given by

$$
U=\frac{\hat{\omega}+m+\Gamma_{(2 n)}^{i} \hat{p}_{i}}{\sqrt{2 \hat{\omega}(\hat{\omega}+m)}}=\frac{1}{\sqrt{2 \hat{\omega}(\hat{\omega}+m)}}\left(\begin{array}{cc}
(\hat{\omega}+m) & \sigma_{(2 n)}^{i} p_{i} \\
-\sigma_{(2 n)}^{i} p_{i} & (\hat{\omega}+m)
\end{array}\right)
$$

connects the Dirac and Foldy- Wouthuysen representations. Using the explicit expressions for $B_{F T}$ and the properties (29) of $\Lambda$ matrices we obtain:

$$
B_{F W} \equiv B_{F T}
$$

Using (36) we see, that in the space-time dimensions where the Majorana particle exists, namely when $D=2,4(\bmod 8)$, the following relation holds (with appropriate choice of $\beta$ ) :

$$
B_{F W}=B_{Q M}
$$

and hence the quantum mechanical description of the neutral particle in the spacetime dimensions $D=2,4(\bmod 8)$ coincides with the field theoretical description of the Majorana particle in the Foldy- Wouthuysen representations.

Note again, that in quantum mechanics the neutral particle exists in any even dimensions $D=2 n$.

\section{$3 \quad D=2 n-$ dimensional Weyl particle}

Consider a theory with the action given by the expression

$$
\begin{aligned}
S & =\frac{1}{2} \int d \tau\left[\frac{1}{e}\left(z^{\mu}\right)^{2}+e m^{2}-i\left(\xi_{\mu} \dot{\xi}^{\mu}-\xi_{D+1} \dot{\xi}_{D+1}\right)+i m \chi \xi_{D+1}\right] \\
z^{\mu} & =\dot{x}^{\mu}-\frac{i}{2} \chi \xi^{\mu}-\frac{(-i)^{\frac{D-2}{2}}}{(D-2) !} \varepsilon^{\mu \nu \lambda_{1} \ldots \lambda_{D-2}} b_{\nu} \xi_{\lambda_{1}} \cdots \xi_{\lambda_{D-2}}+\tilde{\alpha} b^{\mu}
\end{aligned}
$$

Here $b^{\mu}$ are additional fields, $\tilde{\alpha}$ is a constant $; b^{\mu}, \tilde{\alpha}$ are Grassmann even. 
This theory is gauge invariant under the transformations: the reparametrization transformations

$$
\begin{aligned}
& \delta x^{\mu}=u \dot{x}^{\mu}, \quad \delta e=\frac{d}{d \tau}(u e), \quad \delta b^{\mu}=\frac{d}{d \tau}\left(u b^{\mu}\right), \\
& \delta \xi^{\mu}=u \dot{\xi}^{\mu}, \quad \delta \chi=\frac{d}{d \tau}(u \chi), \quad \delta \xi_{D+1}=u \dot{\xi}_{D+1}
\end{aligned}
$$

with the even parameter $u(\tau)$, supergauge transformations

$$
\begin{aligned}
\delta x^{\mu} & =i v \xi^{\mu}, \quad \delta e=i v \chi, \quad \delta b^{\mu}=0, \quad \delta \xi^{\mu}=v \frac{z^{\mu}}{e}, \quad \delta \chi=2 \dot{v}, \quad \delta \xi_{D+1}=v m \\
z^{\mu} & =\dot{x}^{\mu}-\frac{i}{2} \chi \xi^{\mu}-\frac{(-i)^{\frac{D-2}{2}}}{(D-2) !} \varepsilon^{\mu \nu \lambda_{1} \ldots \lambda_{D-2}} b_{\nu} \xi_{\lambda_{1}} \cdots \xi_{\lambda_{D-2}}+\tilde{\alpha} b^{\mu}
\end{aligned}
$$

with the odd parameter $v(\tau)$.

Let us find the equations of motion, corresponding to the variations of the action over $e$ and $b_{\mu}$ :

$$
\begin{aligned}
& p_{\mu}^{2}-m^{2}=0, \quad p_{\mu} \equiv \frac{1}{e} z_{\mu}, \\
& \frac{(-i)^{\frac{D-2}{2}}}{(D-2) !} \varepsilon^{\mu \nu \lambda_{1} \ldots \lambda_{D-2}} p_{\nu} \xi_{\lambda_{1}} \cdots \xi_{\lambda_{D-2}}+\tilde{\alpha} p^{\mu}=0 .
\end{aligned}
$$

Multiplying the second of these equations by $p_{\mu}$ we find the condition

$$
\tilde{\alpha} m^{2}=0
$$

Thus the theory with the action (41) is not contradictory only for the choice of the parameters $\tilde{\alpha}=0$ or (when $\tilde{\alpha} \neq 0$ ) $m=0$. In what follows we will consider the case of $\tilde{\alpha} \neq 0$, hence we must put $m=0$ in (41). Thus consider a theory with the action given by the expression

$$
S=\int d \tau\left[\frac{1}{2 e}\left(\dot{x}^{\mu}-\frac{i}{2} \chi \xi^{\mu}-\frac{(-i)^{\frac{D-2}{2}}}{(D-2) !} \varepsilon^{\mu \nu \lambda_{1} \ldots \lambda_{D-2}} b_{\nu} \xi_{\lambda_{1}} \cdots \xi_{\lambda_{D-2}}+\tilde{\alpha} b^{\mu}\right)^{2}-\frac{i}{2} \xi_{\mu} \dot{\xi}^{\mu}\right]
$$

which is a generalization to the space-time dimension $D=2 n$ of the pseudoclassical theory of Weyl particle [23] (it turns out, that the variable $\xi_{D+1}$ can be omitted from the action (41) at $m=0$ ). Apart from the invariance under the transformations (42) and (43) the action (46) is invariant under the additional gauge transformations 23

$$
\delta x^{\mu}=\frac{(-i)^{\frac{D-2}{2}}}{(D-2) !} \varepsilon^{\mu \nu \lambda_{1} \ldots \lambda_{D-2}} \eta_{\nu} \xi_{\lambda_{1}} \cdots \xi_{\lambda_{D-2}}-\tilde{\alpha} \eta^{\mu}
$$




$$
\begin{aligned}
\delta \xi^{\mu} & =\frac{1}{e} \frac{(-i)^{\frac{D}{2}}}{(D-3) !} \varepsilon^{\mu \nu \delta \lambda_{2} \ldots \lambda_{D-2}} \eta_{\nu} z_{\delta} \xi_{\lambda_{2}} \cdots \xi_{\lambda_{D-2}}, \\
\delta b^{\mu} & =\frac{d}{d \tau}\left(\eta^{\mu}\right), \quad \delta \chi=-2 \eta_{\nu}\left(p^{\nu} \xi^{\sigma}-p^{\sigma} \xi^{\nu}\right) b_{\sigma} \delta_{D 4}, \quad \delta e=-2 i \eta_{\nu} \xi^{\nu} \xi^{\sigma} b_{\sigma} \delta_{D 4}
\end{aligned}
$$

with the even parameter $\eta_{\nu}(\tau)$.

Acting in the standard way we obtain the canonical hamiltonian of the theory, which is given by the expression

$$
\begin{aligned}
H & =\dot{x}^{\mu} p_{\mu}+\dot{\xi}^{\mu} \pi_{\mu}-L= \\
& =\frac{e}{2} p^{2}+\frac{i}{2} \chi p_{\mu} \xi^{\mu}-\left(\frac{(-i)^{\frac{D-2}{2}}}{(D-2) !} \varepsilon^{\nu \mu \lambda_{1} \ldots \lambda_{D-2}} p_{\mu} \xi_{\lambda_{1}} \cdots \xi_{\lambda_{D-2}}+\tilde{\alpha} p^{\nu}\right) b_{\nu}
\end{aligned}
$$

primary constraints

$$
\Phi_{1}^{(1)}=\pi_{e}, \quad \Phi_{2}^{(1)}=\pi_{\chi}, \quad \Phi_{3 \mu}^{(1)}=\pi_{\mu}-\frac{i}{2} \xi_{\mu}, \quad \Phi_{4 \mu}^{(1)}=\pi_{\mu}^{b}
$$

and the secondary constraints

$$
\begin{aligned}
\Phi_{1}^{(2)} & =\left|p_{0}\right|-\omega, \quad \Phi_{2}^{(2)}=p_{\mu} \xi^{\mu} \\
\Phi_{3 \mu}^{(2)} & \equiv T_{\mu}=\frac{(-i)^{\frac{D-2}{2}}}{(D-2) !} \varepsilon_{\mu \nu \lambda_{1} \ldots \lambda_{D-2}} p^{\nu} \xi^{\lambda_{1}} \cdots \xi^{\lambda_{D-2}}+\tilde{\alpha} p_{\mu}
\end{aligned}
$$

where $\omega=|\vec{p}|, \vec{p}=\left(p_{k}\right), k=1, \ldots, D-1$. One can see now, that the canonical hamiltonian $H$ is equal to zero on the constraints surface, as it was expected to be.

The constraints $F \equiv\left(\Phi_{1}^{(1)}, \Phi_{2}^{(1)}, \Phi_{4 \mu}^{(1)}, \Phi_{1}^{(2)}\right), \Phi_{3 \mu}^{(2)}$ are first class. Apart from them there is one more first class constraint $\varphi$, which is a linear combination of the constraints $\Phi_{3 \mu}^{(1)}, \Phi_{2}^{(2)}$ :

$$
\varphi=p^{\mu} \Phi_{3 \mu}^{(1)}+i \Phi_{2}^{(2)}=p_{\mu} \pi^{\mu}+\frac{i}{2} p_{\mu} \xi^{\mu}
$$

Adhering to the quantization method, when already at the classical level all gauge degrees of freedom are fixed [16], we must introduce into the theory additional constraints, equal in number to that of all first class constraints and conjugated to the latter. However, as it was noted in [23], the constraints $\Phi_{3 \mu}^{(2)}$ for $D \geq 4$ are at least quadratic functions of the variables $\xi^{\lambda}$, thus complicating the introduction of additional constraints conjugated to $\Phi_{3 \mu}^{(2)}$. For this reason the constraints $\Phi_{3 \mu}^{(2)}$ after quantization will be used as conditions 
on the state vectors. For the remaining first class constraints $F$ and $\varphi$ we will introduce additional constraints $\Phi^{G}$ in the form

$$
\Phi_{1}^{G}=x^{0}-\kappa \tau, \quad \Phi_{2}^{G}=\chi, \quad \Phi_{3}^{G}=e-\frac{\kappa}{p_{0}}, \quad \Phi_{4 \nu}^{G}=b_{\nu}, \quad \Phi_{5}^{G}=\xi^{0} .
$$

To go over to time-independent set of constraints we perform a canonical transformation (9), after which the hamiltonian of the system on the constraint surface is given by the expression

$$
H=\omega=|\vec{p}|
$$

The quantization of the theory is carried out using the formulae of the previous section with $m=0$.

Note, that in comparison with the previous section, the system of second-class constraints in this theory contains new constraints $\Phi_{4 \mu}^{(1)}=\pi_{\mu}^{b} \approx 0, \Phi_{4 \mu}^{G}=b_{\mu} \approx 0$. However they have a special form [27] and they do not affect the final Dirac brackets (the variables $b_{\mu}$ and $\pi_{\mu}^{b}$ can be excluded from the theory using the constraints).

Now using the expressions (12) for $\hat{\xi}^{i}$ with $m=0$, we can find the expressions for the operators $\hat{T}_{\mu}$, which correspond to the first class constraints $\Phi_{3 \mu}^{(2)}$ :

$$
\hat{T}_{\mu}=\left(\frac{\hbar}{2}\right)^{(D-2) / 2} \hat{p}_{\mu} \hat{T}, \quad \hat{T}=\hat{\kappa} \frac{\hat{p}^{i} \Sigma^{i}}{\hat{\omega}}-\alpha, \quad \hat{p}_{0}=-\hat{\kappa} \hat{\omega}, \quad \alpha=\left(\frac{\hbar}{2}\right)^{-(D-2) / 2} \tilde{\alpha} .
$$

To deduce these relations we used the equality $\varepsilon_{01 \ldots(D-1)}=-\varepsilon_{12 \ldots(D-1)}=-1$, and also the relation

$$
\frac{(-i)^{\frac{D-2}{2}}}{(D-2) !} \varepsilon_{j_{1} \cdots j_{D-2}}^{i} \sigma^{j_{1}} \cdots \sigma^{j_{D-2}}=\sigma^{i}
$$

from the $\sigma^{i}$-matrix algebra in $(D-1)$-dimensional space [29]. The $\alpha$ is the eigenvalue of the chirality operator $\hat{\vec{p}} \vec{\sigma} / \hat{\omega}$ and in quantum mechanics can have the values +1 or -1 .

As it was already mentioned above, the operators $\hat{T}_{\mu}$ will be used to impose conditions on the physical state vectors. For the $D$-dimensional space-time the state vector has in general $2^{D / 2}$ components (in massless case this representation is reducible). Representing the state vector $f$ in the form

$$
f=\left(\begin{array}{c}
f^{1} \\
f^{2}
\end{array}\right)
$$


where $f^{1}$ and $f^{2}$ are $2^{(D-2) / 2}$ dimensional columns, we write down the equations for the state vector in the form:

$$
\hat{T} f=0
$$

It is natural to interpret the quantum mechanics constructed above as a theory of Weyl particle in the Foldy-Wouthuysen representation. Indeed, using the realization (13) for $\hat{\kappa}$ we write the operator $\hat{T}$ in the form

$$
\hat{T} \equiv \hat{T}_{F W}=\Gamma_{(2 n)}^{0} \frac{\hat{p}^{i} \Sigma^{i}}{\hat{\omega}}-\alpha
$$

Consider also the Shrödinger equation $(i \partial / \partial \tau-\hat{H}) f=0$, which describes the evolution of the state-vector $f$ with respect to parameter $\tau$. Being rewritten in terms of the physical time $x^{0}=\kappa \tau$ it takes the form

$$
\left(i \partial / \partial x^{0}-\Gamma_{(2 n)}^{0} \hat{\omega}\right) f=0
$$

Applying the unitary Foldy-Wouthuysen transformation for $m=0$ in $D$ dimensional space-time

$$
f=U \psi, \quad U=\frac{\hat{\omega}+\Gamma_{(2 n)}^{i} \hat{p}^{i}}{\hat{\omega} \sqrt{2}}
$$

where $\psi$ is the wave function in the Dirac representation, we find [16, 17] that the Shrödinger equation transforms into Dirac equation, the expressions for the Lorentz generators $\hat{J}^{\mu \nu}$ transform into standard expressions for the Lorentz generators in the Dirac representation. Furthermore, by direct calculation one can prove that the operator $\hat{T}_{F W}$ transforms into the $\hat{T}_{D}$ operator

$$
\hat{T}_{D}=U^{+} \hat{T}_{F W} U=\Gamma_{(2 n)}^{D+1}-\alpha
$$

which is proportional to a standard Weyl projector in the Dirac representation.

Thus we see that the quantum mechanical description constructed here after the Foldy-Wouthuysen transformation turns into the Dirac description of the Weyl particle. Hence the quantum mechanics constructed above describes the Weyl particle in the Foldy-Wouthuysen representation. 


\section{Quantum mechanics of Majorana-Weyl particle}

As it was discussed in section 2 , the invariance of the action under the reparametrization transformation $\tau \rightarrow-\tau$ allows, after the introduction of this transformation in the gauge group, to describe a neutral particle in the QM. We will apply this ideology to the action (46) of the previous section.

For the invariance of the action with respect to the transformation $\tau \rightarrow-\tau$ apart from (18) we need a transformation

$$
b^{\mu}(\tau) \rightarrow-b^{\mu}(-\tau)
$$

Now the action (46) will be invariant under the transformations (18), (62) if

$$
\frac{D-2}{2}=2 k
$$

i.e. in the space-time dimensions $D=4 k+2$. In these dimensions we will quantize the action (46) with the extended gauge group and will compare this result with the description of Majorana-Weyl particle in the field theory. Adhering to the first approach to quantization, when instead of the gauge $\Phi=x^{0}-\kappa \tau$ we choose the $\Phi^{\prime}=x^{0}-\tau$ gauge, we simply restrict the physical states space to the sector $\kappa=+1$ of the Weyl particle in QM. The wave function is described now by a $2^{(D-2) / 2}$ column

$$
f=f^{1},
$$

while the Weyl condition has the form

$$
\hat{P}_{\alpha} f^{1} \equiv \frac{1}{2}\left(\frac{\hat{\vec{p}} \vec{\sigma}}{\hat{\omega}}-\alpha\right) f^{1}=0,
$$

where the operators $\hat{P}_{\alpha}$ is defined by relation

$$
\hat{P}_{\alpha}=\left.\frac{1}{2} \hat{T}\right|_{\kappa=+1}
$$

For the comparison with the field theory it is convenient to introduce the "bispinor" $f_{M}$

$$
f_{M}=\left(\begin{array}{c}
f^{1} \\
\Lambda f^{1 *}
\end{array}\right)
$$


The same wave function turns out in the second approach to quantization, when we factorize the total states space of the Weyl particle with respect to the gauge symmetry $\tau \rightarrow-\tau$. Only now one more condition must be fulfilled

$$
\hat{P}_{-\alpha} \Lambda f^{1 *}=0,
$$

from which follows that the relation

$$
\hat{P}_{-\alpha} \sim \Lambda \hat{P}_{\alpha}^{*} \Lambda^{-1}
$$

must hold. Using the explicit formulae (24), (30) and the properties (29) of $\Lambda$-matrices we convince ourselves, that this relation is true only for dimensions $D=4 k+2$. Thus two approaches to quantization give equivalent answers: the quantum mechanics in space-time dimensions $D=4 k+2$ with enlarged gauge group describes a neutral (the antiparticle coincides with the particle) Weyl particle. Since this description can be equivalent to the field theoretical description of the Majorana particle only when $D=8 k+2$ and $D=8 k+4$, we find, that in the dimensions $D=8 k+2$ the constructed QM is equivalent to field theoretical description of Majorana-Weyl particle in the Foldy-Wouthuysen representation. The singling out of the dimensions $D=8 k+2$ is in agreement with the fact, that in the field theory Majorana-Weyl particle exists only in these dimensions [28] (in QM the neutral Weyl particle exists also in dimensions $D=8 k+6$ ).

This research was partially supported by the grants RFBR/INTAS-96-829 and 2115291 YPI of the German Bundesministerium für Forschung und Technologie. I.V.Tyutin was supported in part by grant \# M21300 from international Science Foundation and Government of the Russian Federation and by European Community Commission under contract INTAS-94-2317.

\section{References}

[1] Berezin F.A. and Marinov M.S. JETP.Lett., 21 (1975) 321.

[2] F.A.Berezin and M.S. Marinov. Ann. Phys., 104 (1977) 336.

[3] R.Casalbuoni. Nuovo Cimento, A33 (1976) 115. 
[4] A. Barducci, R. Casalbuoni, and L. Lusanna. Nuovo Cimento, A35 (1976) 377.

[5] L. Brink, S. Deser, B. Zumino, P. Di Vecchia, and P. Howe. Phys. Lett., B64 (1976) 435.

[6] L. Brink, P. Di Vecchia, and P. Howe. Nucl. Phys., B118 (1977) 76.

[7] A.P.Balachandran, P.Salomonson, B.S.Skagerstam, and J.O.Winnberg. Phys. Rev., 15(8) (1977) 2308.

[8] A. Barducci, R. Casalbuoni, and L. Lusanna. Phys.Lett. 64B (1976) 319.

[9] A. Barducci, R. Casalbuoni, D.Dominici and L. Lusanna. Phys.Lett. 100B (1981) 126.

[10] A. Barducci and L. Lusanna. Nuovo Cimento, A77 (1983) 39.

[11] A. Barducci and L. Lusanna. Journ. Phys, A16 (1983) 1993.

[12] P. Howe, S. Penati, M. Pernici, and P. Townsend. Phys. Rev., B215 (1988) 555.

[13] P. Howe, S. Penati, M. Pernici, and P. Townsend. Class. Quant. Grav., 6 (1989) 1125.

[14] J. Gomis, M. Novell, and K. Rafanelli. Phys. Rev., D34 (1986) 1072.

[15] Gershun V.D. and Tkach V.I. JETP.Lett., 29 (1979) 320.

[16] Gitman D.M and Tyutin I.V. Class. Quant. Grav., 7 (1990) 2131.

[17] Grigoryan G.V. and Grigoryan R.P. Yad. Phys., 53 (1991) 1737.

[18] A.A. Zheltukhin. Theor. Math. Phys., 65 (1985) 1072.

[19] Gitman D.and Saa A. Class. Quant. Grav.., 10 (1993) 1447.

[20] Grigoryan G.V. and Grigoryan R.P. Yad. Phys., 58 (1995) 1.

[21] Grigoryan G.V., Grigoryan R.P. and Sarkissian G.A. Yad. Phys., 59 (1996) 552 
[22] Cortes J.L. and Plyushchay M.S. and Velazquez L. Phys.Lett., 306 (1993) 34.

[23] Gitman D.M, Gonçalves A.E., and Tyutin I.V. Phys.Rev., D34 (1994) 5439.

[24] Grigoryan G.V., Grigoryan R.P. and Tyutin I.V. Preprint YERPHY-1446(16)95:hep-th/-9510002, Yad. Phys., (in press)

[25] M.B.Green, J.H.Schwartz, and E.Witten. Superstring theory. Cambridge University Press, Cambridge, 1987.

[26] P.A.M.Dirac. Lectures on Quantum Mechanics. Yeshiva University, N.Y., 1964.

[27] Gitman D.M. and Tyutin I.V. Quantization of fields with constraints. Springer Verlag, Berlin, 1990.

[28] J.Sherk. Extended supersymmetry and extended supergravity theory. In M.Levy and S.Deser, editors, Recent Development in Gravitation.

[29] Case K.M. Phys. Rev., 97 (1955) 810. 